\section{Creating Pseudocolor Images using ImageJ}

Joel B. Sheffield

Department of Biology, Temple University, Philadelphia PA jbs@temple.edu

It is common for microscopists to examine fluorescent samples that have been exposed to a mixed set of fluorophores with specific staining properties. Images are often captured from these samples as a set, using different filter sets to isolate specific elements of the sample. Sometimes the images are captured in gray scale, and in other cases, they are captured with color cameras. In either case, one would often like to combine the images to create a single image that presents the information from all fluorophores at once. This kind of display is particularly useful for determining the relative locations of different components.

The public domain program, ImageJ, written by Wayne Rasband at the National Institutes of Health, Bethesda, MD. (http://rsb. info.nih.gov/ij/), and extensively supplemented by many contributors, contains a simple routine for taking three gray scale images, assigning a separate color to each and merging them into an RGB pseudocolor image.

The process starts with the set of three images such as the following, which are derived from buccal cells stained with Wheat Germ Agglutinin-Rhodamine, MitoTracker-Green, and DAPI, respectively.
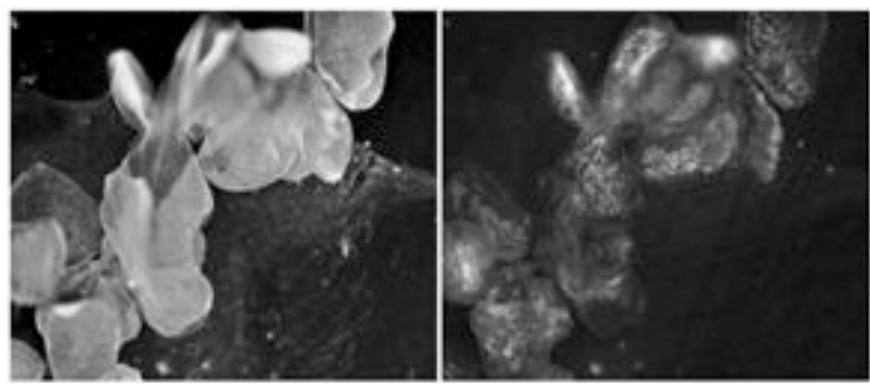

Figure 1

The three pictures are opened individually in ImageJ. Then, the menu item "Image $>$ color $>$ RGB merge" is selected.

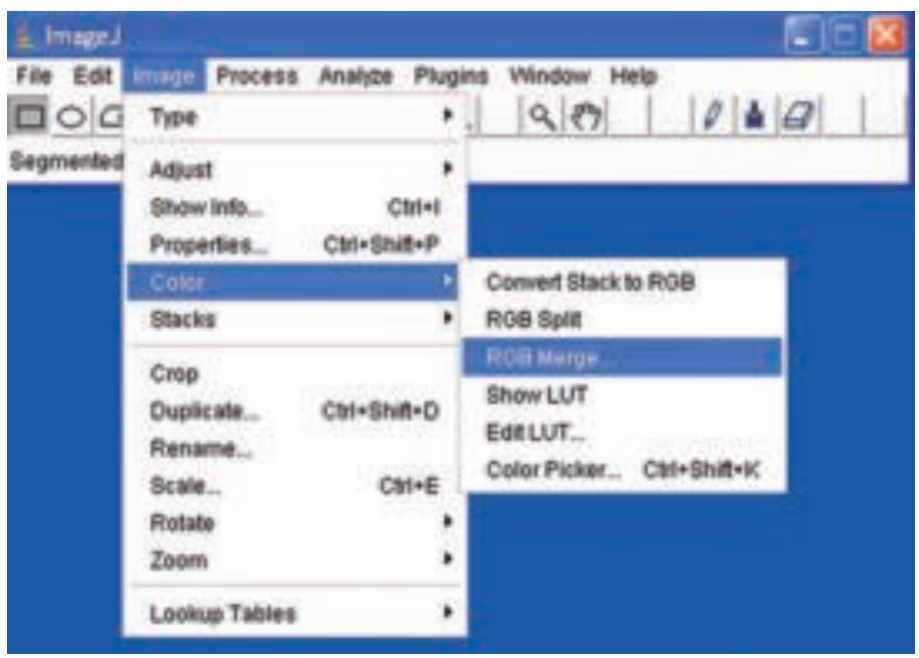

Figure 2

This brings up a dialog box that allows one to enter the specific images that are to form the final product.

Note that one has the option to enter any of the pictures in any of the channels. The most direct is to put each image into the ap- propriate channel. That is, red to red, green to green, etc. It is generally useful to check the "Keep source images" box for further experimentation. After clicking on "OK", the RGB image is generated (Figure 4).

It is also possible to manipulate the channels for special purposes. As an example, the

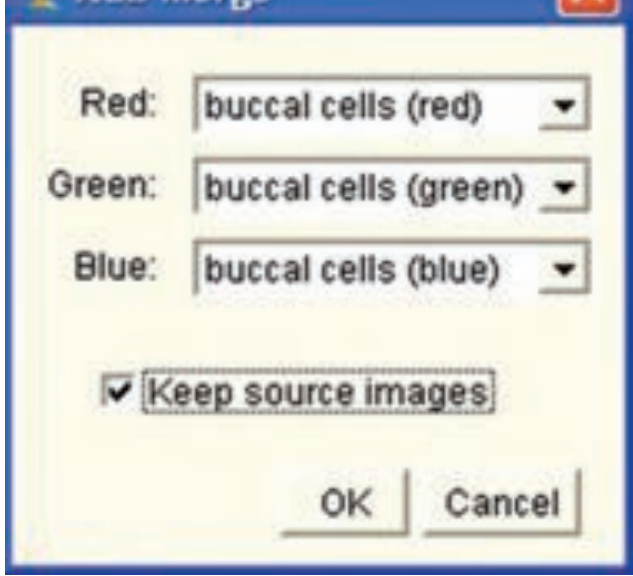

Figure 3 image from the red source was used for both the red and the blue channels, (omitting the blue data) to create an image of magenta and green (Figure 5). This display is particularly useful for those viewers who are deuteranopes (red/green color blind).

Once the image is formed, it is possible to adjust each of the color channels independently using the menu item "Image $>$ Adjust $>$ Color Balance"

There are several constraints that must be considered when

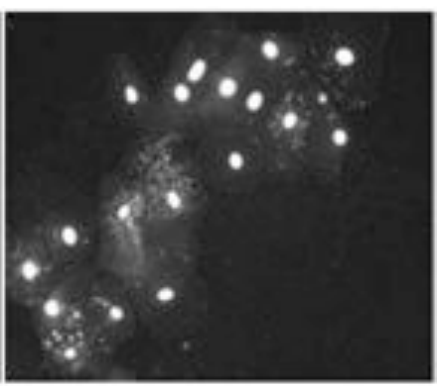
preparing such images.

First, the separate images need to be aligned. Unfortunately, ImageJ does not yet have the ability to superimpose layers that could be moved independently, as in Photoshop. On the other hand, there is a registration plugin available that allows either manual or automatic alignment prior to image assembly.

Second, it is important that the intensity distribution in each of the images be within the 8-bit dynamic range. This avoids oversaturation in the final image.

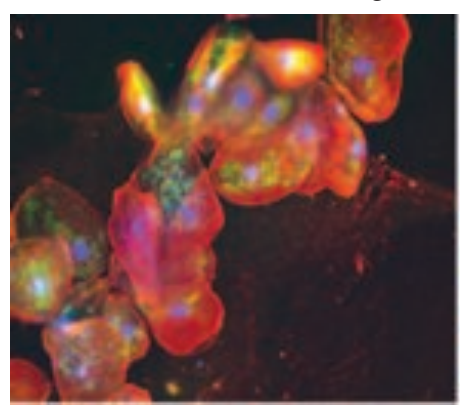

Figure 4

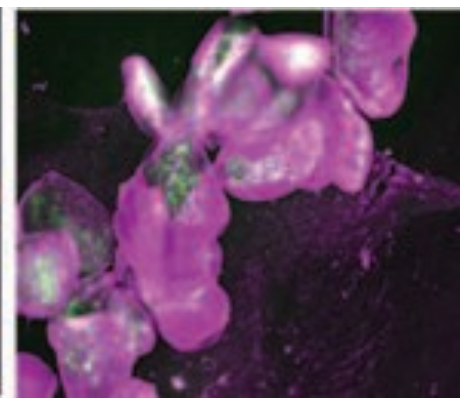

Figure 5
Third, it should be realized that images such as these are transforms from the original data. Each color channel activates a single type of pixel in the display. As a result, some of the subtlety of imaging is lost. DAPI, for instance has a significant fluorescence in the green wavelengths, which is lost with this kind of image, but might be retained in a color original.

Editor's Note: The author and Tony Collins, McMaster Univ, will be chairing a session on ImageJ at M\&M-2007 in Fort Lauderdale. 


\section{Advanced}

\section{Detector}

Technology

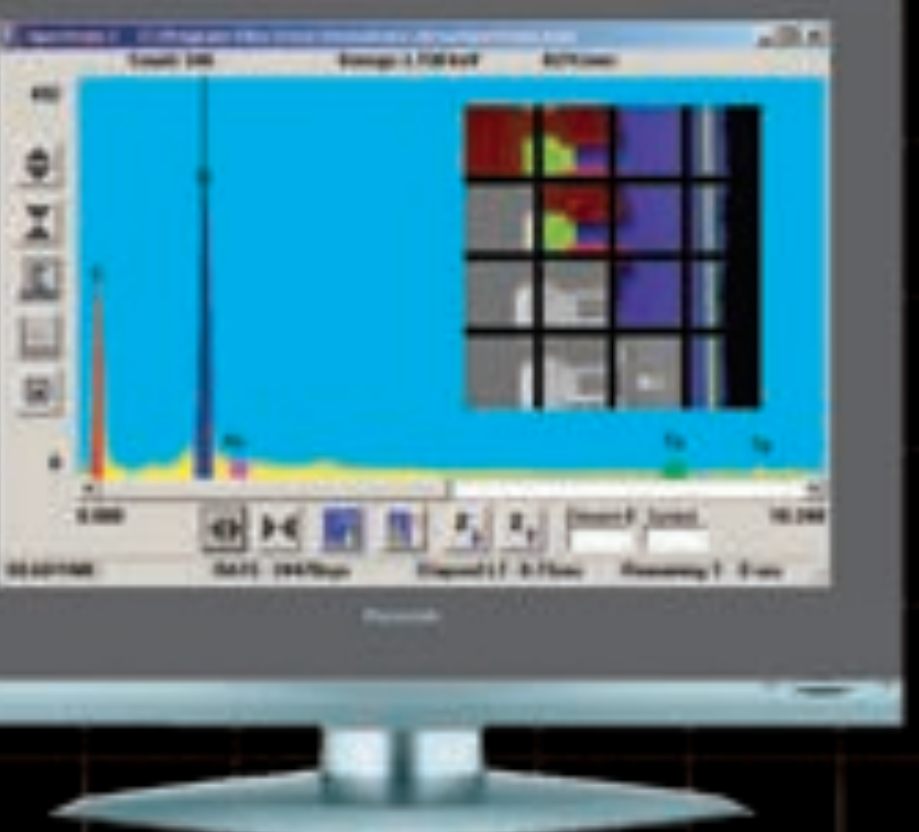

Spectral Acquisition

Digital Imaging

Elemental Mapping

Indexed Mapping

\section{NanoAnalysis for Electron Microscopy}

Evex

www.evex.com
857 State Road Princeton, NJ 08540 609-252-9192 Telephone $609-252-9091 \mathrm{Fax}$ 